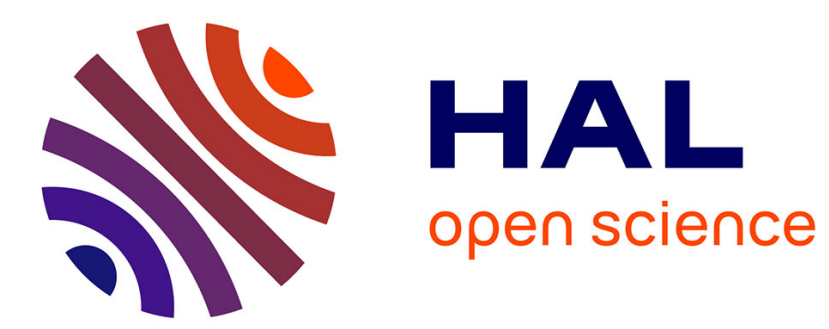

\title{
SHORT-RANGE ORDER IN PAIRS AND TETRAHEDRON CLUSTERS OF A 50 at \% B.C.C. BINARY SOLID SOLUTION
}

\author{
U. Gahn
}

\section{- To cite this version:}

U. Gahn. SHORT-RANGE ORDER IN PAIRS AND TETRAHEDRON CLUSTERS OF A 50 at \% B.C.C. BINARY SOLID SOLUTION. Journal de Physique Colloques, 1977, 38 (C7), pp.C7-378-C7381. 10.1051/jphyscol:1977775 . jpa-00217278

\section{HAL Id: jpa-00217278 https://hal.science/jpa-00217278}

Submitted on 1 Jan 1977

HAL is a multi-disciplinary open access archive for the deposit and dissemination of scientific research documents, whether they are published or not. The documents may come from teaching and research institutions in France or abroad, or from public or private research centers.
L'archive ouverte pluridisciplinaire HAL, est destinée au dépôt et à la diffusion de documents scientifiques de niveau recherche, publiés ou non, émanant des établissements d'enseignement et de recherche français ou étrangers, des laboratoires publics ou privés. 


\title{
SHORT-RANGE ORDER IN PAIRS AND TETRAHEDRON CLUSTERS OF A 50 at \% B.C.C. BINARY SOLID SOLUTION
}

\author{
U. GAHN \\ Max-Planck-Institut für Eisenforschung GmbH, Düsseldorf West-Germany
}

\begin{abstract}
Résumé. - Un alliage binaire équiatomique est traité avec trois modèles statistiques différents, ceux de Bragg-Williams-Gorsky, Kikuchi et Cowley. Le modèle de Cowley donne l'information des probabilités de paires tandis que le modèle de Kikuchi fournit en plus des probabilités de clusters. Par le modèle de Bragg-Williams-Gorsky les probabilités de paires et de clusters peuvent être déterminées à partir des probabilités de points mais seulement approximativement. Les données reportées en forme de diagrammes peuvent servir de guide pour des investigations expérimentales futures.
\end{abstract}

\begin{abstract}
An equiatomic binary b.c.c. alloy is treated by three different statistical models, those of Bragg-Williams-Gorsky, Kikuchi, and Cowley. The Cowley model gives information about pair probabilities in different coordination shells, while the Kikuchi model describes tetrahedron cluster probabilities in addition to that. In the Bragg-Williams-Gorsky model pair and tetrahedron probabilities can be determined only approximately from point probabilities. The reported data in form of diagrams can serve as a background information for future experimental investigations.
\end{abstract}

1. Introduction. - Two previous papers $[1,2]$ dealt with short-range order (s.r.o.) and long-range order (l.r.o.) in f.c.c. and b.c.c. binary solid solutions. The calculations based on different statistical models, especially on those of Bragg-Williams-Gorsky (B.W.G.), Kikuchi (K.), and Cowley (C.). A comprehensive review article concerning these models has been written by Domb [3].

Here a special case has been picked up again, i.e. a 50 at $\%$ b.c.c. solid solution of homogeneous concentration distribution. This case appears easy to survey, even if s.r.o. of higher than second neighbours or s.r.o. of clusters is considered. The model treatment for other compositions, as done in [2] and shown for first and second neighbour pair probabilities, leads to data and diagrams which especially for clusters can only be badly arranged and in principle give no new information.

As in [2] the data are calculated on the basic assumption that only first and second neighbour interactions $W^{(1)}, W^{(2)}>0\left({ }^{1}\right)$ need be taken into account. The further restriction, $W^{(1)}=2 W^{(2)}$, will hold, too.

2. Nomenclature. - This representation follows the version in [2], and so the symbols used there are adopted here. The s.r.o. parameters $p_{\mathrm{AB}}^{(i)}, i=1,2, \ldots$, are the values of the pair probabilities to find A-B-

( $\left.{ }^{1}\right)$ The energy parameters $W^{(i)}=V_{A A}^{(i)}+V_{B B}^{(i)}-2 V_{A B}^{(i)}, i=1$, 2 , expressed in terms of pair potentials, are considered to be independent of temperature and composition. atom pairs, the atoms sitting at the $i$-th neighbour distance of each other :

$$
p_{\mathrm{AB}}^{(i)}=N_{\mathrm{AB}}^{(i)} /\left(N z^{(i)} / 2\right) .
$$

$N_{\mathrm{AB}}^{(i)}$ is the corresponding number of pairs, $N$ the total number of lattice sites, and $z^{(i)}$ the coordination number.

The quadruplet probability for tetrahedron clusters penetrating two elementary b.c.c. cells (see figures 3 or 4) consists of the number $N_{\mathrm{I} J \mathrm{~K} \mathrm{~L}}^{\mathrm{II} \mathrm{HIV}}$ (divided by $6 N$ ) of quadruplets IJKL with the I (=A or B)-atom sitting on sublattice $\mathrm{I}$, the $\mathrm{J}(=\mathrm{A}$ or $\mathrm{B}$ )-atom sitting on sublattice II, etc... The normalizing denominator $6 \mathrm{~N}$ gives the total number of tetrahedra. The division of the crystal lattice into four sublattices - primarily introduced for the definition of 1.r.o. parameters $\left({ }^{2}\right)-$ has been done as usual, i.e. in such a manner that atoms of the sublattices I and II (second neighbours among one another) are first neighbours to atoms of III and IV (second neighbours among one another, too).

3. Discussion of the Diagrams. - Figure 1 represents the s.r.o. situation in the first and second shell. This is already known (cf. [2]) and gives the starting

(2) The 1.r.o. parameter (point probability) $p_{A}^{L}=N_{A}^{L} /(N / 4)$, $L=\mathrm{I}, \ldots, \mathrm{IV}$, is the number $N_{\mathrm{A}}^{L}$ of A-atoms on sublattice $L$, referred to the number of all the sublattice sites; similarly $p_{B}^{L}=1-p_{A}^{L}$; $\sum_{L} p_{A}^{L}=4 c_{A} ; c_{A}$ is the atomic fraction of $A$-atoms. Above a critical temperature $T_{c}$ it is : $p_{\mathrm{A}}^{L}=c_{\mathrm{A}}, p_{\mathrm{B}}^{L}=c_{\mathrm{B}}$. 


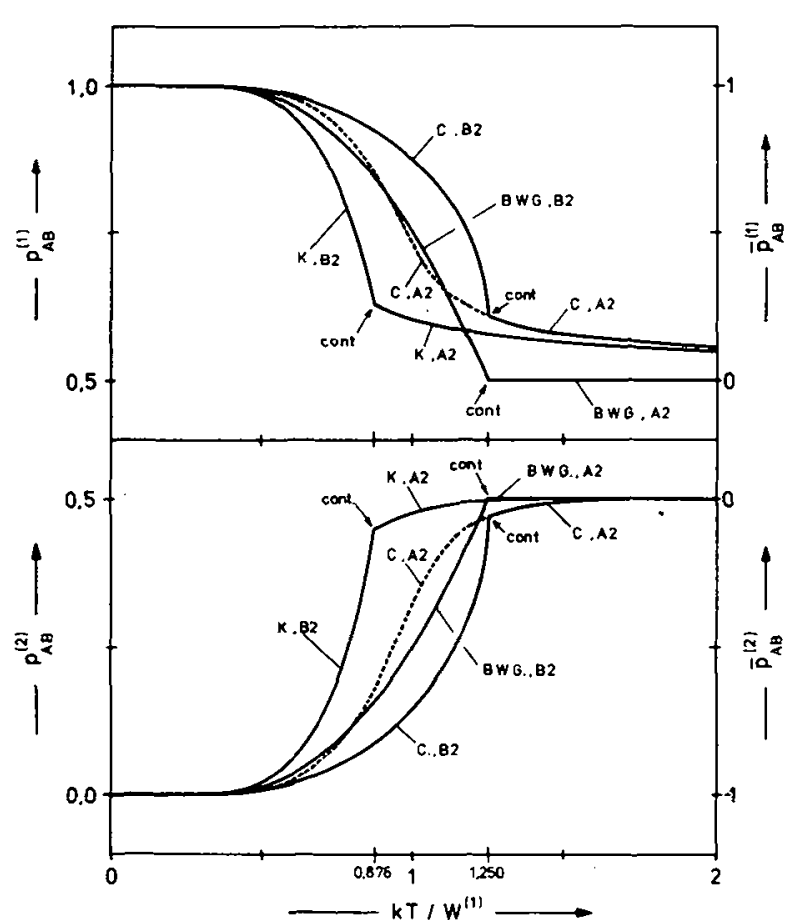

FIG. 1. - Pair probability in the B.W.G., K., and C. model ("cont.": continuous transitions at $T_{\mathrm{c}}$; dashed curve parts : no long-range order included in the model calculations).

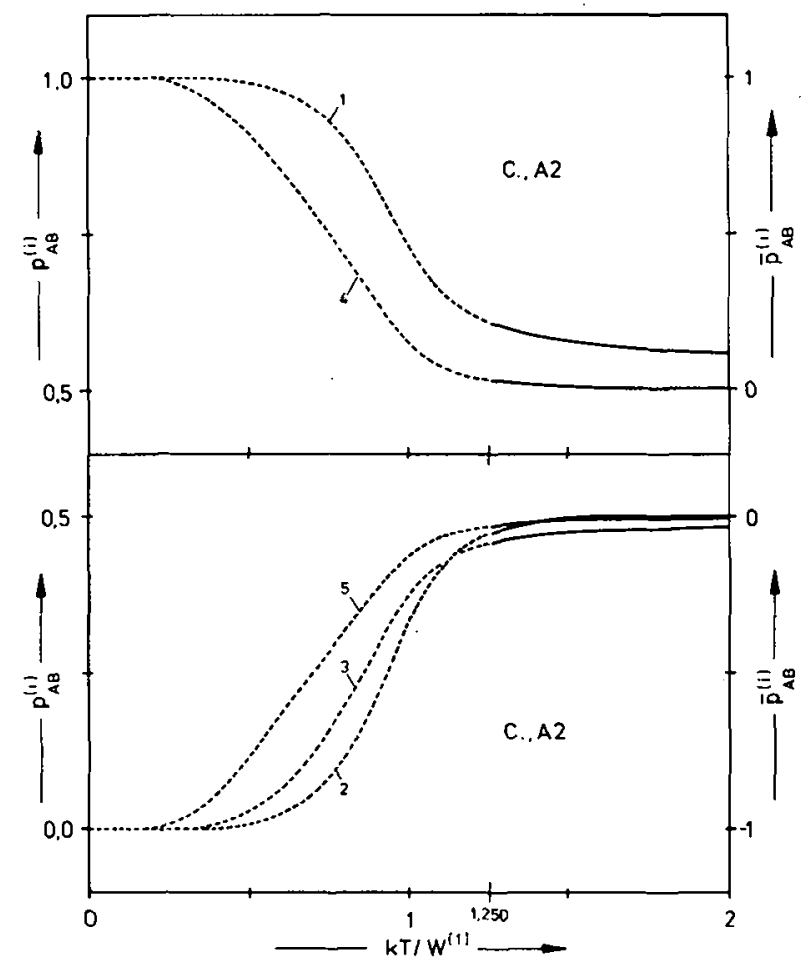

FIG. 2. - Pair probability up to the fifth shell in the C. model (dashed curve parts : cf. fig. 1).

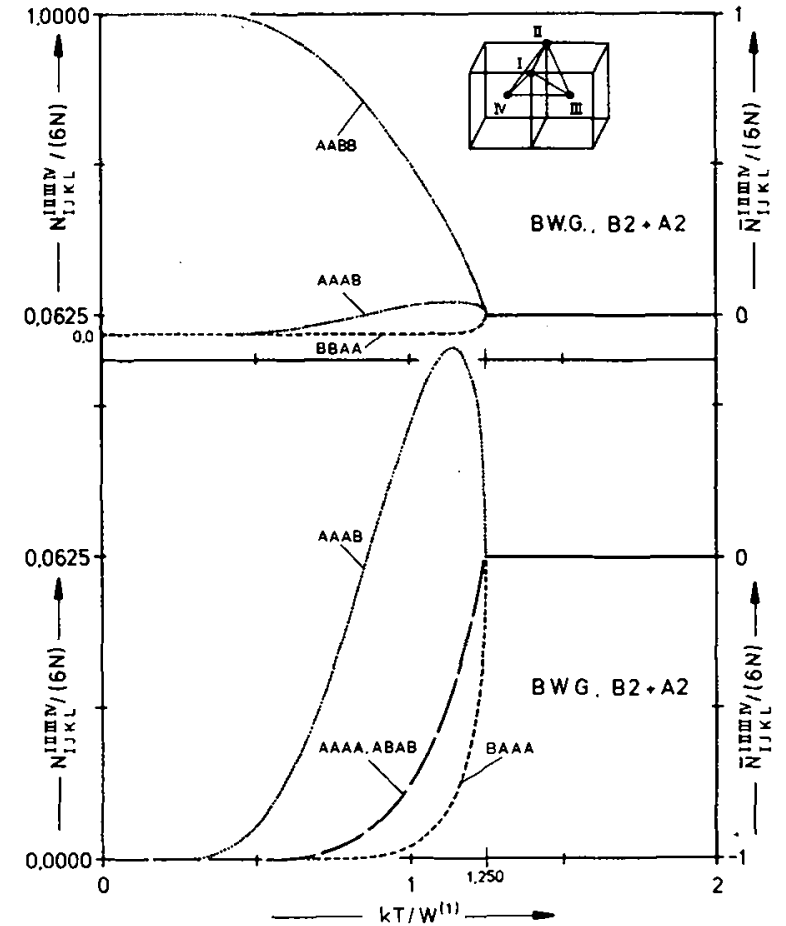

FIG. 3. - Tetrahedron probability in the B.W.G. model (position of cluster sites depicted).

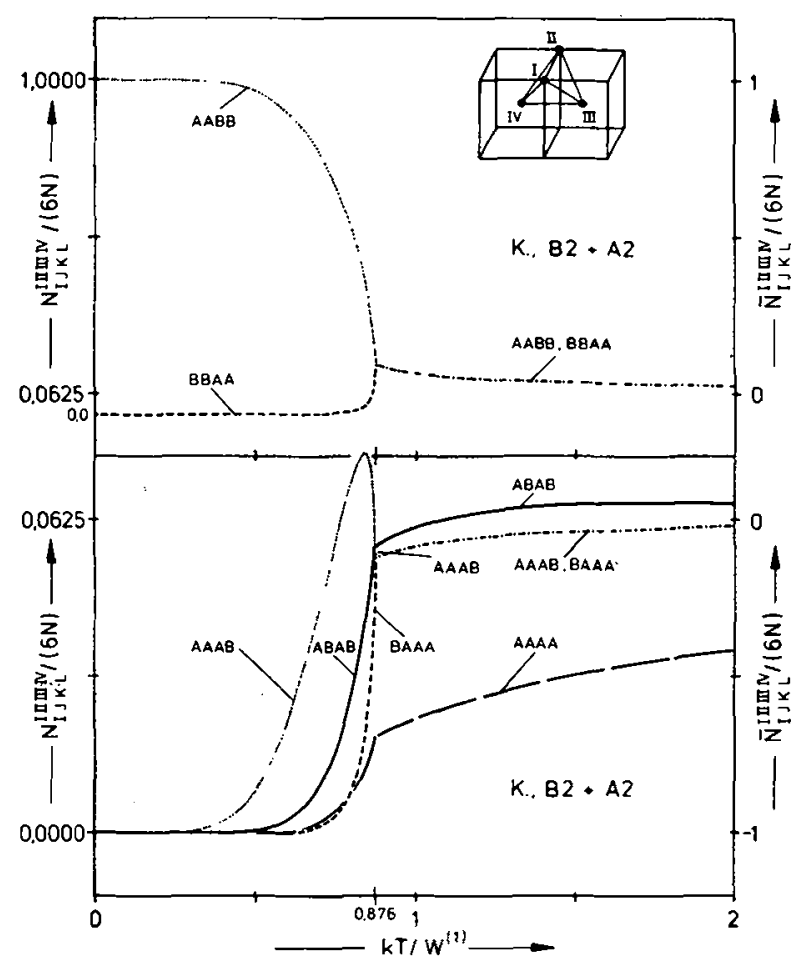

Fig. 4. - Tetrahedron probability in the K. model.

Short-range order parameters as pair probabilities $p_{A B}^{(i)}, i=1, \ldots, 5$ (normalized $\left.\bar{p}_{A B}^{(i)}\right)$ and tetrahedron probabilities $N_{1 j}^{\text {III K } \mathrm{L}} / /(6 N)($ normalized $\tilde{N}_{1}^{11}$ III L $\left./(6 N)\right) v s$. normalized temperature $k T / W^{(1)}$. Results for three different models (with interactions in two coordination shells : $\left.W^{(2)} / W^{(1)}=1 / 2\right):$ Bragg-Williams-Gorsky (B.W.G.), Kikuchi (K.) and Cowley (C.). The 50 at \% binary b.c.c. crystal is long-range ordered (B2) or not (A2). 
point of the present discussion. The s.r.o. parameters $p_{\mathrm{AB}}^{(1)}$ and $p_{\mathrm{AB}}^{(2)}$ are shown as functions of the temperature for three different models, i.e. of B.W.G., K. and C. At the critical temperature $T_{\mathrm{c}}=0.876 \mathrm{~W}^{(1)} / \mathrm{k}$ for the $K$. model (tetrahedron approximation) or $T_{\mathrm{c}}=1.250 \mathrm{~W}^{(1)} / \mathrm{k}$ for the B.W.G. and C. models there are continuous (cont.) transitions from the 1.r.o. crystal state (B2-structure $\left.\left({ }^{3}\right)\right)$ to the disordered (i.e. without 1.r.o.) state (A2-structure $\left({ }^{3}\right)$ ).

The B.W.G. S.r.o. data have been won on the assumption that the pair probability can approximately be got from products of point probabilities, i.e. from the 1.r.o. parameters of two different sublattices (cf. [2]). Thus in the B.W.G. model already just above $T_{\mathrm{c}}=1.250 \mathrm{~W}^{(1)} / k$ the limiting value $2 c_{\mathrm{A}} c_{\mathrm{B}}$ is reached.

Concerning the $\mathrm{C}$. model the dashed curve part and the consequent fully drawn part above $T_{\mathrm{c}}$ describe a crystal with s.r.o. but without l.r.o. That is the result of the original $\mathrm{C}$. model, which primarily does not include any 1.r.o. But similarly to the procedure in the B.W.G. model now in the inverse direction, in the $C$. model the 1.r.o. has been introduced via the s.r.o. parameters. Taking 1.r.o. into account in this manner the full curve part indicated by « $\mathrm{C}$., B2 » is found [2].

In the B.W.G. model the way to find higher shell probabilities $p_{\mathrm{AB}}^{(i)}, i>2$, is simple, thinking on their determination by products of 1.r.o. parameters. It turns out that $p_{\mathrm{AB}}^{(3)}=p_{\mathrm{AB}}^{(5)}=p_{\mathrm{AB}}^{(2)} ; p_{\mathrm{AB}}^{(4)}=p_{\mathrm{AB}}^{(1)} ;$ etc. (cf. eq. (2) in [2]) $\left(^{4}\right)$.

The C. model demands an explicit calculation of all the $p_{\mathrm{AB}}^{(i)}$; for details see [2]. Figure 2 represents the results for $i=1, \ldots, 5$. As in figure 1 the curve sections at $T<T_{\mathrm{c}}$ are dashed, the effect of 1.r.o. has not been shown. Stress here is laid on the mutual relation of s.r.o. values of different shells. Beginning at $T=0 \mathrm{~K}$ with complete s.r.o., which means complete 1.r.o. in any case, then increasing the temperature the s.r.o. values slowly tend to the final values $2 c_{A} c_{B}$, with $p_{\mathrm{AB}}^{(3)}, p_{\mathrm{AB}}^{(4)}, p_{\mathrm{AB}}^{(5)}$ more rapidly decreasing (or increasing) as $p_{\mathrm{AB}}^{(1)}, p_{\mathrm{AB}}^{(2)}-$ only near below and above $T_{\mathrm{c}}$ there is a little bit more s.r.o. in the third than in the second shell. The dominance of $p_{A B}^{(1)}$ and $p_{A B}^{(2)}$ rests on the inclusion of non-vanishing energy parameters $W^{(i)}$ in the first and second shell. The additional account of l.r.o. raises $p_{\mathrm{AB}}^{(4)}$ nearly up to $p_{\mathrm{AB}}^{(1)}$ (i.e. «C., B2 » in figure 1, upper part) - with a maximum difference of about $20 \%$ at $T_{\mathrm{c}}$ and a difference smaller than $5 \%$ below $k T / W^{(1)}=1.1-$ and it makes $p_{\mathrm{AB}}^{(3)}, p_{\mathrm{AB}}^{(5)}$ coincide with $p_{\mathrm{AB}}^{(2)}$ (i.e. « C., B2 » in figure 1 , lower part) within an error of less than $2 \%$.

$$
\begin{aligned}
& \text { ( } \left.{ }^{3}\right) \text { B2-structure : } p_{\mathrm{A}}^{\mathrm{I}}=p_{\mathrm{A}}^{\mathrm{II}} \neq p_{\mathrm{A}}^{\mathrm{III}}=p_{\mathrm{A}}^{\mathrm{IV}} \\
& \mathrm{DO}_{3} \text {-structure : } p_{\mathrm{A}}^{\mathrm{I}}=p_{\mathrm{A}}^{\mathrm{II}} \neq p_{\mathrm{A}}^{\mathrm{III}} \neq p_{\mathrm{A}}^{\mathrm{IV}} \text { or } p_{\mathrm{A}}^{\mathrm{I}}=p_{\mathrm{A}}^{\mathrm{II}}=p_{\mathrm{A}}^{\mathrm{III}} \neq p_{\mathrm{A}}^{\mathrm{IV}} \text {. } \\
& \text { A2-structure : } p_{\mathrm{A}}^{\mathrm{I}}=p_{\mathrm{A}}^{\mathrm{II}}=p_{\mathrm{A}}^{\mathrm{II}}=p_{\mathrm{A}}^{\mathrm{IV}} \text {. }
\end{aligned}
$$

$\left({ }^{4}\right)$ In the $\mathrm{K}$ model (tetrahedron approximation) this is the only possible way, too, but the results are inconsistent with the directly calculated values of $p_{A B}^{(1)}, p_{A B}^{(2)}$. of course.
An idea of s.r.o. in clusters shall be conveyed by the figures 3 and 4 . A special cluster, a tetrahedron, has been chosen - suitable for the treatment in the $\mathrm{K}$. model. The normalized quadruplet numbers $N_{\text {I J K L III }}^{\text {III }}$ are plotted versus the same abscissa as in the preceding figures.

At first in figure 3 the B.W.G. model is used as before, that means $N_{1 \mathrm{JKL}}^{\mathrm{IIIIV}} /(6 N)$ is replaced by $p_{\mathrm{I}}^{\mathrm{I}} p_{\mathrm{J}}^{\mathrm{II}} p_{\mathrm{K}}^{\mathrm{III}} p_{\mathrm{L}}^{\mathrm{IV}}$. (A corresponding procedure in the $\mathrm{C}$. model, constructing tetrahedra from pairs, is not unique and has not been performed.) One of the two «B2-clusters », $\mathrm{AABB}$ and $\mathrm{BBAA}$, reminding of the corresponding 1.r.o. structure, is clearly prevailing at lower temperatures. The lower diagram of figure 3 retakes a small section of the upper ordinate between 0 and $\left(c_{\mathrm{A}}\right)^{4}=\left(c_{\mathrm{B}}\right)^{4}=0.0625$. Thus another cluster, $\mathrm{AAAB}$, which can be called a « $\mathrm{DO}_{3}$-cluster $»$, reveals a distinct rounded peak near below $T_{\mathrm{c}}$.

For the $\mathrm{K}$ model a similar situation is found in figure 4 , even the peak turns out though not so strongly developed. So the cluster variation method of Kikuchi gives a probability value for the $\mathrm{DO}_{3}$ cluster, which increasing from zero surpasses the limiting value $\left(c_{\mathrm{A}}\right)^{4}$, too, and then - before $T$ reaches $T_{\mathrm{c}}$ - sinks down to a value hardly smaller than $\left(c_{\mathrm{A}}\right)^{4}$.

This result is surprising in so far that no. 1.r.o. $\mathrm{DO}_{3}$-structure exists at $c_{\mathrm{A}}=0.5$, yet it is principally prepared by the s.r.o. It reminds of the fact, shown in figure 3 in [2], that for $c_{\mathrm{A}}=0.75 \ll \mathrm{B} 2$-pairs » appear (characterized by the value $p_{\mathrm{AB}}^{(2)}<2 c_{\mathrm{A}} c_{\mathrm{B}}$ for $\left.T_{\mathrm{c}} \leqslant T \leqslant 0.63 W^{(1)} / k\right)$, though no $\mathrm{B} 2$-structure is predicted by the $K$. model at $c_{A}=0.75$ (and $\left.W^{(2)}=W^{(1)} / 2\right)$. Little deviations from the stœechiometric compositions will bring forth the 1.r.o. structures already announced by the s.r.o.

A further s.r.o. feature can be noted, namely the curve of the AAAA-cluster. This cluster, indicating a sort of miniature segregation, has particularly low probability values for $T>T_{\mathrm{c}}$, the absolute difference to $\left(c_{\mathrm{A}}\right)^{4}$ being comparable to those of the B2-clusters $\mathrm{AABB}$ and BBAA.

For the sake of completeness it should be noted that in principle there are sixteen different tetrahedron clusters, but the probabilities for some of them are identical if $c_{\mathrm{A}}=0.5$. Keeping to the sublattice sequence I, II, III, IV six clusters with the following site population can be distinguished :
1) $\mathrm{AAAA}=\mathrm{BBBB}$,
2) $\mathrm{AAAB}=\mathrm{AABA}=\mathrm{ABBB}=\mathrm{BABB}$,
3) $\mathrm{BAAA}=\mathrm{ABAA}=\mathrm{BBBA}=\mathrm{BBAB}$,
4) $\mathrm{ABAB}=\mathrm{ABBA}=\mathrm{BABA}=\mathrm{BAAB}$,
5) $\mathrm{AABB}$,
6) BBAA .

In the case of the B.W.G. model the probabilities of type 1) and 4) coincide. 
4. Conclusions. - The usefulness of the statistical models concerning the interpretation of experimental data is hard to test, because it is still difficult to get reliable experimental s.r.o. data e.g. from X-ray measurements in view of the many necessary corrections for the separation of other but s.r.o. effects, which reach the same order of magnitude. Especially for b.c.c. alloys there is a lack of profound experimental data $[4,5]$. Considering the underlying models it follows that the $\mathrm{K}$. model directly faces the problem of determining cluster probabilities (points, pairs, triangles, and tetrahedra, coupled in a self-consisting manner - greater clusters are also possible), while the B.W.G. or C. model gives data principally only of point or pair probabilities resp., which other proba- bilities follow from on additional assumptions. On the other side the $\mathrm{C}$. model offers a relatively less cumbersome numerical tool for the calculation of even higher than fifth shell pair probabilities. Especially for the inclusion of higher neighbour interactions the amount of numerical labour increases rapidly in the $\mathrm{K}$. model but remains tolerable in the $\mathrm{C}$. model. Thus the choice of the model also depends on the numerical effort; the reliability cannot be checked. The data may only serve as a background information for future experimental information.

Acknowledgments. - Thanks are due to Prof. W. Pitsch for critical discussions.

\section{References}

[1] GaHN, U., Phys. Status Solidi (a) 29 (1975) 529.

[2] Gahn, U., Phys. Status Solidi (a) 40 (1977) 463.

[3] Doмв, C., Adv. Phys. 9 (1960) 149.

[4] Order-Disorder Transformation in Solids, Proc. Internat. Symp., Tubingén, 1973, Ed. : H. Warlimont (Springer-Verlag, Berlin) 1974.
[5] Internat. Discussion Meeting on Studies of Lattice Distortions and Local Atomic Arrangements by X-Ray, Neutron and Electron Diffraction, Jülich, $1974 ; J$. Appl. Crystallogr. 8 (1975) 79-344. 